\title{
An Update on Efficacy and Safety of Emerging Hepatic Antifibrotic Agents
}

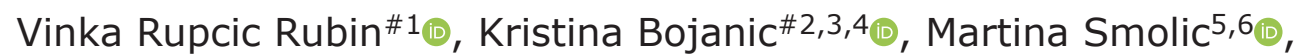 \\ Jurica Rubin7, Ashraf Tabl|8,9 and Robert Smolic*7,10,11
}

${ }^{1}$ Department of Gynaecology and Obstetrics, Osijek University Hospital Centre, Faculty of Medicine, Josip Juraj Strossmayer University of Osijek, Osijek, Croatia; ${ }^{2}$ Department of Biophysics and Radiology, Faculty of Dental Medicine and Health Osijek, J. J. Strossmayer University of Osijek, Osijek, Croatia; ${ }^{3}$ Department of Biophysics and Radiology, Faculty of Medicine Osijek, J. J. Strossmayer University of Osijek, Osijek, Croatia; ${ }^{4}$ Department of Radiology, Health Center Osijek, Osijek, Croatia; ${ }^{5}$ Department of Pharmacology and Biochemistry, Faculty of Dental Medicine and Health Osijek, J. J. Strossmayer University of Osijek, Osijek, Croatia; ${ }^{6}$ Department of Pharmacology, Faculty of Medicine Osijek, J. J. Strossmayer University of Osijek, Osijek, Croatia; 'Department of Medicine, Division of Gastroenterology/Hepatology, University Hospital Osijek, Osijek, Croatia; 8 Microbial Biotechnology Department, Genetic Engineering and Biotechnology Research Division, National Research Centre, Dokki, Egypt; ${ }^{9}$ Department of immunology, Egypt Center for Research and Regenerative; Medicine (ECRRM), Cairo, Egypt; ${ }^{10}$ Department of Pathophysiology, Physiology and Immunology, Faculty of Dental Medicine and Health

Osijek, J. J. Strossmayer University of Osijek, Osijek, Croatia; ${ }^{11}$ Department of Pathophysiology, Faculty of Medicine Osijek, J. J. Strossmayer University of Osijek, Osijek, Croatia

\begin{abstract}
Liver fibrosis represents a response to chronic liver injury. Metabolic dysfunction-associated fatty liver disease and metabolic dysfunction-associated steatohepatitis are the most common chronic liver diseases, both with increasing incidence. Therefore, there is a great impetus for development of agents targeting these conditions. Accumulating data on possible treatment options for liver fibrosis are emerging in the literature. However, despite extensive research and much effort in the field, approved agents for liver fibrosis are still lacking. In this critical review, we have summarized the main data about specific treatment options for liver fibrosis gained from ongoing clinical trials, with an emphasis on efficacy and safety of these agents.
\end{abstract}

Citation of this article: Rupcic Rubin V, Bojanic K, Smolic M, Rubin J, Tabll A, Smolic R. An update on efficacy and safety of emerging hepatic antifibrotic agents. J Clin Transl Hepatol 2021;000(000):000-000. doi: 10.14218/JCTH.2020.00040.

Keywords: Liver fibrosis; MAFLD; MASH; Clinical trials; Antifibrotic agents; Efficacy; Safety.

Abbreviations: YGT, gamma-glutamyl transferase; ACC, acetyl-coenzyme A carboxylase; ALT, alanine aminotransferase; ARB, angiotensin receptor blocker; ASK1, apoptosis signal-regulating kinase; AST, aspartate aminotransferase; CCR, C-C motif chemokine receptor; DAA, direct-acting antiviral; FGF, fibroblast growth factor; FXR, farnesoid X receptor; GLP, glucagon-like peptide; HCV, hepatitis $C$ virus: HIV human immunodeficiency virus: IgA, Immunoglobulin $A$ IL interleukin; LOXL2, lysyl oxidase-like homolo $\mathrm{IL}$, interleukin; $\mathrm{LOXL2}$, lysyl oxidase-like hotabolic dys function-associated fatty liver disease; $M A S H$, metabolic dysfunction-associated steatohepatitis; MC, mast cell; MR, magnetic resonance; OCA, obeticholic acid; PBC, primary biliary cholangitis; PPARs, peroxisome proliferator-activated receptors; PSC, primary sclerosing cholangitis; SCD1, stearoyl COA desaturase 1 ; T2DM, type 2 diabetes mellitus; THR, thyroid hormone receptor; UDCA, ursodeoxycholic acid; VAP-1, vascular adhesion protein-1.

Received: 03 May 2020; Revised: 22 November 2020; Accepted: 9 December 2020

\#These authors contributed equally to this work.

*Correspondence to: Robert Smolic, J. J. Strossmayer University of Osijek Faculty of Medicine Osijek, Department of Pathophysiology J. Huttlera 4, Osijek 31000, Croatia. Tel: +385-31-512-800, Fax: +385-31-512-833, E-mail: rsmolic@mefos.hr

\section{Introduction}

Liver fibrosis represents a pathogenic response to chronic liver injury. ${ }^{1}$ Among the etiologies, viral hepatitis B and $\mathrm{C}$ infections have been historically cited as the most common causes. In the last 30 years, certain changes in prevalence have occurred as a consequence of the spread of Western lifestyle and therapeutic advances in the treatment of some liver diseases. Intriguingly, while the prevalence of chronic hepatitis B and alcoholic liver disease has remained stable, the prevalence of chronic hepatitis $C$ has decreased. Along with the increasing prevalence of type 2 diabetes mellitus (T2DM), obesity and metabolic syndrome, prevalence of metabolic dysfunction-associated fatty liver disease (MAFLD) has notably increased. ${ }^{2}$ MAFLD, ${ }^{3}$ in particular, has become the most common form of chronic liver disease, with global prevalence of $25.4 \%,{ }^{4}$ and a major cause of liver cirrhosis and hepatocellular carcinoma. ${ }^{3}$ Other, less common etiologies include cholestatic disease (primary biliary cholangitis (PBC) and primary sclerosing cholangitis (PSC)), autoimmune hepatitis and such genetic diseases as hemochromatosis, alpha antitrypsin deficiency, and cystic fibrosis. ${ }^{4}$

However, regardless of the cause, a common and the most prominent feature of all advanced chronic liver diseases is liver fibrosis. ${ }^{2}$ At the histopathological level, liver fibrosis represents qualitative and quantitative changes in extracellular matrix and deposition of type I collagen, primarily, which ultimately results in disorganization of the liver parenchyma architecture. ${ }^{4}$ Fibrosis stage is the strongest predictor of prognosis in patients with chronic liver diseases, linked not only to liver-related but also to extrahepatic morbidity and mortality. ${ }^{5}$

During the last decades, basic science studies have investigated the underlying molecular and pathophysiological mechanisms of liver fibrosis in detail, aiming to explore potential new drug targets. Currently, at least six groups of fundamental pathophysiological mechanisms of liver fibrosis development could be distinguished (Table 1) and consequently treatment strategies can be differentiated as treatments of the underlying disease(s), targeting cell death, liver metabolism, gut-liver axis, fibrogenesis (in the 
Rupcic Rubin V. et al: Antifibrotic agents: an update

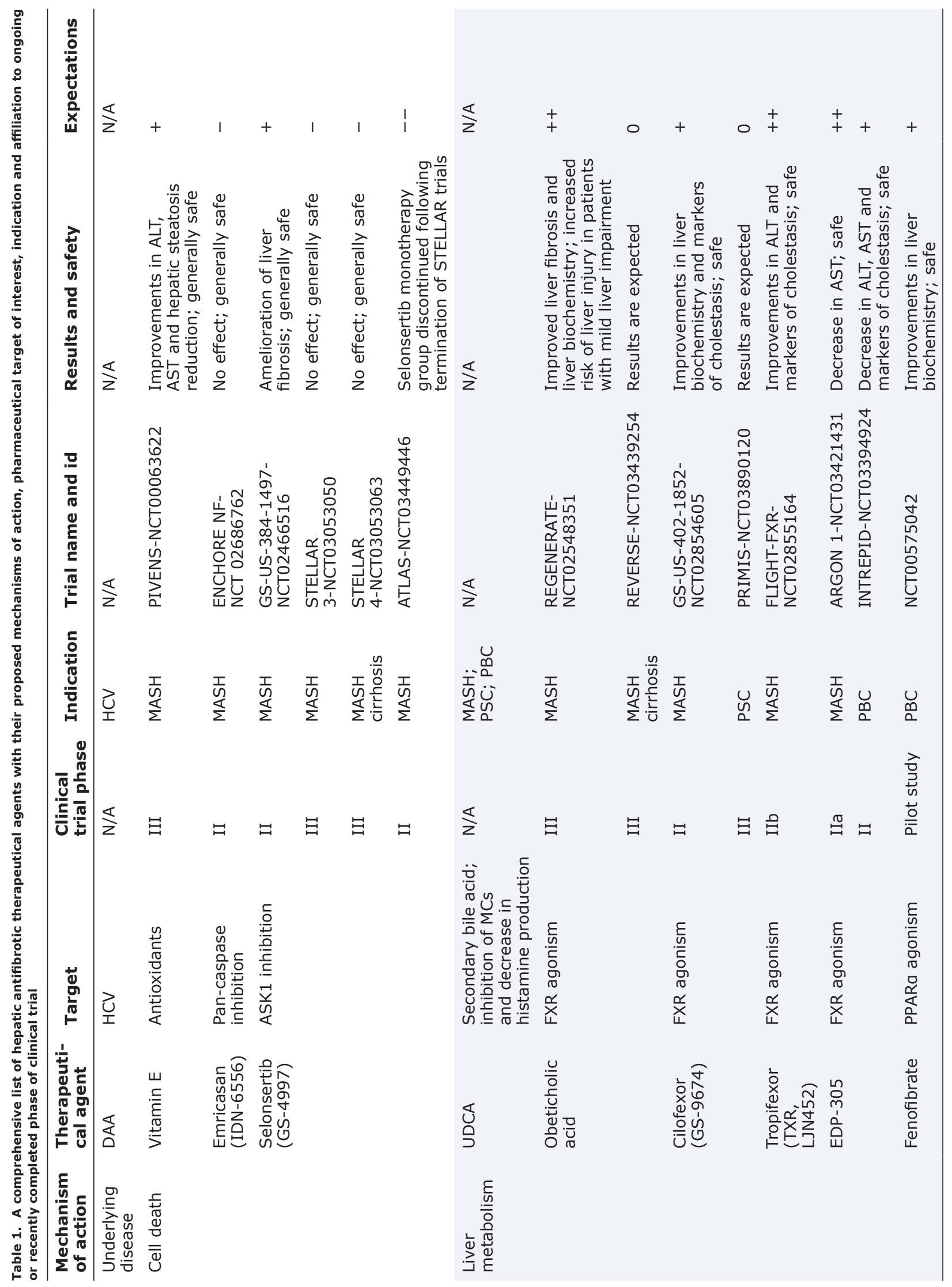


Rupcic Rubin V. et al: Antifibrotic agents: an update

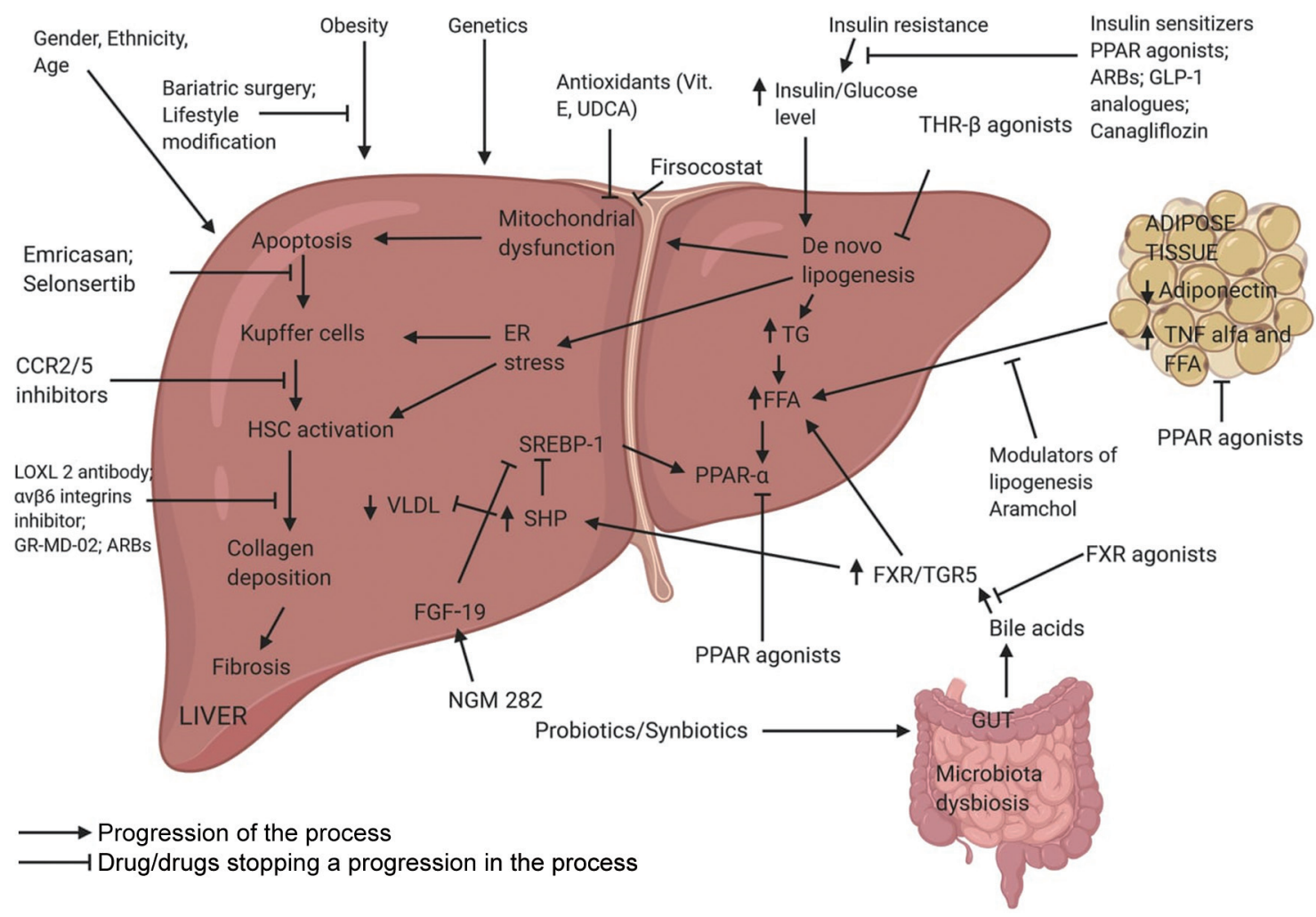

Fig. 1. Treatments for metabolic dysfunction-associated fatty liver disease (MAFLD) and metabolic dysfunction-associated steatohepatitis (MASH) according to mechanism of action. Abbreviations: ARB, angiotensin receptor blocker; CCR2/5, C-C motif chemokine receptor; ER, endoplasmic reticulum; FFA, free fatty acids; FXR, farnesoid X receptor; GLP, glucagon-like peptide; HSC, hepatic stellate cell; LOXL 2, lysyl oxidase-like homolog 2; PPAR, peroxisome proliferatoractivated receptor; SHP, small heterodimer partner; SREBP, sterol regulatory element binding protein; TG, triglycerides; TGR5, Takeda G protein-coupled receptor 5; THR, thyroid hormone receptor; TNF, tumor necrosis factor; UDCA, ursodeoxycholic acid; VLDL, very low-density lipoprotein.

narrow sense of the word), and inflammation.

In this critical review, we will present the latest findings on underlying molecular and pathophysiological mechanisms in the development of liver fibrosis and their utilization as potential therapeutic targets, as shown in Fig. 1. The boundaries between aforementioned pathways are arbitrary and in vivo these pathways overlap continuously. Although clinical trials have examined the effectiveness of lifestyle changes, bariatric surgery and hepatitis C and B suppression, however, the same is out of the scope of this review. This review focuses on pharmaceuticals; particularly, we will highlight data about efficacy and safety of these potential drugs gained from the currently ongoing or recently completed phases 2 and 3 clinical trials.

\section{Treatment options for different pathophysiological mechanisms of liver fibrosis development}

\section{Treatments of the underlying disease}

Although cirrhosis is considered to be a definitive and irreversible stage of hepatic fibrosis, several studies have shown that the cure or suppression of hepatitis C and B can result in regression of hepatic fibrosis and even cirrhosis. 6,7 Specific etiologic treatments for chronic hepatitis $B$ and $C$ that have resulted in sustained virologic response have also been associated with decreased fibrosis and cirrhosis regression. Similarly, lifestyle changes or bariatric surgery achieving weight loss in patients with metabolic dysfunction-associated stea- tohepatitis (MASH) resulted in reduction of the histological features of MASH and fibrosis reversal. 8,9

Therefore, any treatment for liver fibrosis would ideally include treatment of the underlying disease as well. However, this is not always possible, and also, on the other hand, in some cases of known and cured-cause of chronic liver disease, the persistence of fibrosis or even its progression can still be present.9,10 One possible explanation for that paradox might be that beyond a certain limit, the fibrogenetic process gains a certain relative autonomy. ${ }^{11}$ In such cases, etiologic treatment cannot eliminate life-threatening complications. Therefore, regardless of the etiological factor, novel antifibrotic drugs represent an utmost need for patients with cirrhosis.

\section{Treatments targeting cell death}

In chronic liver disease, hepatocyte death is a profibrotic trigger. ${ }^{12}$ Moreover, an association between the extent of hepatocyte death and the progression of fibrosis in MAFLD was confirmed. ${ }^{13}$ Thus, it can be assumed that inhibition of cell death pathways constitutes a promising target for future antifibrotic treatments. However, different cell types contribute to profibrotic or antifibrotic actions, respectively. Hepatic stellate cells are a main collagen-producing cells in liver fibrosis and, therefore, their apoptosis in terms of regulated death could be crucial in stopping and reversing fibrosis. ${ }^{14}$ Therefore, the current concept of antifibrotic therapy is cell-type specific. ${ }^{12,14}$

Vitamin $\mathrm{E}$ is a long-known antioxidant agent which could 
Rupcic Rubin V. et al: Antifibrotic agents: an update

act protectively in the liver. ${ }^{15}$ Since it inhibits apoptosis and oxidative stress, it could have a positive and maybe even a curative effect. According to some studies, a therapy with vitamin E could enhance biochemical and histological parameters in MAFLD. 16,17 These results are promising but sparse due to a short-lasting duration of these trials. Also, safety and efficacy of vitamin $\mathrm{E}$ has yet to be evaluated. In the PIVENS (pioglitazone, vitamin E or placebo for nonalcoholic steatohepatitis) trial, vitamin E was investigated as a therapy for MASH. The results were preferable in patients treated with high-dose vitamin E (800 IU/day) for 96 weeks compared to placebo. There was an improvement in some histopathology findings recorded. Improved steatosis and decreased alanine aminotransferase (ALT) and aspartate aminotransferase (AST) levels were found; however, effect on fibrosis was not detected. ${ }^{17,18}$ Although a lot of vitamin $E$ benefits have been confirmed, some studies have indicated that it could raise all-cause mortality, prostate cancer in males older than 50, and hemorrhagic stroke risk. ${ }^{16}$ Metaanalysis by $\mathrm{Xu}$ et al. ${ }^{19}$ confirmed that vitamin E supplementation is promising, with significant improvements in some histological parameters in MASH patients, except fibrosis. Since, the results cannot be generalized, as trials were limited, with a short follow-up duration and a small number of participants, additional large-scale high-quality studies are needed to obtain more comprehensive information on vitamin E supplementation for clinical use.

Apoptosis and production of inflammatory cytokines (interleukin (IL)-1 $\beta$ and IL-18), among others factors, are regulated by caspases and enzymes activated by lipotoxicity. Thus, a more specific therapeutic option could be the inhibition of a pan-caspase enzyme by the use of emricasan, a pan-caspase inhibitor (IDN-6556, PF-03491390, Conatus Pharmaceuticals). Effective blockage of liver injury and fibrosis by emricasan thoroughly inhibiting apoptosis of hepatocytes has been demonstrated in a murine model of MASH. ${ }^{20}$ These data supported the hypothesis that emricasan may be a prospective antifibrotic drug in treatment of MASH. ${ }^{20}$ One week of emricasan treatment ameliorated liver fibrosis, portal hypertension and improved liver function in rats with advanced cirrhosis in a preclinical study in 2019. Since beneficial effects, without any apparent hepatic or systemic adverse effects, were demonstrated with emricasan, further clinical evaluation in the treatment of advanced chronic liver disease was encouraged. ${ }^{21}$ However, in a double-blind, placebo-controlled phase 2 clinical trial (ENCORE-NF, NCT02686762) involving $318 \mathrm{MASH}$ patients with fibrosis (stages F1-F3) after 72 weeks, emricasan did not improve histopathological findings of liver fibrosis in the majority of patients. On the contrary, it may have even worsened it. ALT levels were lowered for short-term; however, it seems that emricasan could have redirected liver cells to a different mechanism of cell death and thus resulted in progression of fibrosis. 22 Persistent normalization of ALT in serum was observed during 72 weeks in a small subset of patients. Therefore, only in a minor subgroup of patients, emricasan may have improved fibrosis, as determined by liver biopsy. Other caspase inhibitors under clinical development, VX-166 (Vertex) and GS-9450 (Gilead) have been reviewed recently. ${ }^{2}$

The third promising pathophysiological pathway is inhibition of the apoptosis signal-regulating kinase 1 (ASK1) preventing activation of the aberrant intracellular cascade pathway, ending in cell death. It has been shown that inhibition of ASK1 resulted in the alleviation of hepatic steatosis in diabetic obese mice (over a decade ago). ${ }^{23}$ Selonsertib (GS-4997), an ASK1 inhibitor, was investigated in rodent model of MASH, with promising results published in 2016 . Improvements in cholesterol, bile acid and lipid metabolism, and a reduction of liver steatosis and fibrosis were shown. ${ }^{24}$ Subsequently, a "pilot trail" with selonsertib was conducted in 72 MASH patients with grades 2 or 3 fibrosis in 2018. This phase 2 trail (NCT02466516) confirmed fibrosis improvement, as determined by liver biopsy and magnetic resonance (MR) elastography. Decrease in liver collagen content as well as less inflammation, and a decreased liver stiffness were found. ${ }^{25}$ Recently selonsertib was investigated in two large phase 3 trials in MASH patients with either advanced fibrosis (STELLAR-3, NCT03053050) or cirrhosis (STELLAR-4, NCT03053063), respectively. However, the primary endpoint after 48 weeks was not met in either study and selonsertib actually exhibited worse results than placebo. 26,27 Nevertheless, selonsertib was included in the phase $2 \mathrm{~b}$ of multicenter, randomized, double-blind, placebo-controlled ATLAS trail (NCT03449446), with acetyl-coenzyme A carboxylase (ACC) inhibitor firsocostat and the farnesoid $X$ receptor (FXR) agonist cilofexor in $395 \mathrm{MASH}$ patients with fibrosis (stage F3) or compensated cirrhosis (F4). ${ }^{28} \mathrm{How}-$ ever, the selonsertib monotherapy group was discontinued after the STELLAR trials termination. ${ }^{29}$

\section{Treatments targeting liver metabolism}

Most of the new pharmacological approaches are aiming to diminish altered hepatic metabolism using targets such as the FXR, the family of peroxisome proliferator-activated receptors (PPARs), ACC, fibroblast growth factor (FGF) 21, or glucagon-like peptide (GLP) 1 agonists. ${ }^{30}$

One of the medications often used in treatment of cholestatic liver disease is ursodeoxycholic acid (UDCA), a secondary bile acid. The proposed mechanism of action of UDCA is decrease of histamine production by mast cells (MCs). MCs enter liver tissue and initiate fibrosis. Thus, inhibition of MCs infiltrating liver might be a potential efficient therapy for PSC. UDCA treatment significantly reduced levels of gamma-glutamyl transferase ( $\mathrm{Y}-\mathrm{GT})$ and total bile acids according to a study from 2017. ${ }^{31}$ Effectiveness of vitamin $E$ and UDCA in MAFLD and in patients without diabetes were compared recently. UDCA was as effective as vitamin E when combined with a specific dietary guidance and lifestyle modification. UDCA can be used as an alternative to vitamin $\mathrm{E}$ in the treatment of $\mathrm{MASH}$, as it has minimal side effects and equal tolerability. ${ }^{32}$ Side effects of UDCA are diarrhea, elevated creatinine, elevated blood glucose, leukopenia, peptic ulcer, skin rash, and thrombocytopenia. 32

FXR is expressed in liver cells and intestine. Actually, it belongs to a nuclear receptor family of transcription factors and as such it regulates bile acid flow. Bile is a toxic substance, but the toxic effect of bile on hepatocytes and bile duct epithelial cells can be minimized physiologically through various mechanisms, such as high apical membrane cholesterol and sphingomyelin content, bile hydration and alkalization, micellar binding of bile acids, mucin formation, and particularly bile flow. ${ }^{33}$ FXR is being explored as a prospective drug for cholestasis, since it could potentially protect liver cells by binding bile acids. When bile acids are bound to FXR, bile acid circulation is diminished and lipids and glucose metabolism are regulated. FXR has an important role in lipid synthesis, and in very low density lipoprotein and triglyceride metabolisms also. Recently, FXR expression was compared in specimens from fibrotic patients with MASH and MAFLD and it was shown that FXR had lower expression in MASH specimens compared to MAFLD, respectively, so it could be hypothesized that FXR might slow liver fibrosis. 34

Obeticholic acid (OCA) is a synthetic bile acid and a potent activator of the FXR. In rodent models of $\mathrm{MASH}$, it improved insulin resistance and altered lipid metabolism, showing positive effects on liver fibrosis. ${ }^{35}$ A preclinical study in 2014 found that cirrhosis complications in the form of decreased portal hypertension may be affected in the 
cirrhotic rat model. ${ }^{36}$ Currently, there is an ongoing randomized global phase 3 study to evaluate the impact of OCA on MASH with fibrosis (REGENERATE, NCT02548351) and on MASH with cirrhosis (REVERSE, NCT03439254), expected to be completed in 2022. After 72 weeks of treatment with $25 \mathrm{mg}$ of OCA, the REGENERATE study showed improved histology and biochemistry of liver fibrosis. ${ }^{37}$ Pruritus was found in $51 \%$ of patients as the most common side effects, and in addition an increase in serum cholesterol levels was found in $17 \%$ of patients (compared to $7 \%$ of patients on placebo). This low-density lipoprotein increase could be resolved by administration of statins but its clinical significance on cardiovascular mortality is still unclear. Pruritus as side effect of OCA was registered earlier in $23 \%$ of patients in the FLINT study in 2015.38 OCA improved the histological features of MASH but its long-term benefits and safety have not been clarified yet. ${ }^{38}$ Malaise, severe pruritus, lowered high-density lipoprotein cholesterol, abdominal pain and discomfort were documented as adverse effects of OCA. According to the Food and Drug Administration, OCA increases the risk of serious and fatal liver injury in patients with moderate to severe liver impairment when dosed more frequently than recommended. It may also increase the risk of liver injury in patients with mild liver impairment receiving the recommended dose. However, the drug is approved as a second-line therapy for PBC. 39

Cilofexor (GS-9674) is a nonsteroidal FXR ligand. It was tested in 2019 in a phase 2 double-blind, placebo-controlled study (NCT02854605) in patients with MASH. Improvements were seen in biochemistry liver function tests and markers of cholestasis. However, the most common side effect recorded was pruritus. Other side effects also reported were rib fracture, diarrhea/dehydration, and acute kidney injury. 40 There is also an ongoing phase 3 trial (NCT03890120, PRIMIS) on 400 patients with PSC. Results are expected in February 2023.41

Tropifexor (LJN-452) is another promising nonsteroidal FXR agonist. According to preclinical research in two distinctive rodent models of MASH, it demonstrated impressive effects in 2019. After 4 weeks of tropifexor therapy, an amelioration of MASH histopathology and reduction in liver triglycerides were observed. Mice that received tropifexor showed dose-dependent reversal of liver fibrosis. ${ }^{42}$ Of course, these promising findings need further evaluation in clinical trials. An active phase 2 trial (NCT02855164) by Novartis Pharmaceuticals (FLIGHT) on 351 patient with MASH was completed. ${ }^{43}$ First results showed amelioration of ALT levels and markers of cholestasis. ${ }^{44}$ According to one study from 2019, which included 95 healthy volunteers, the tolerance was good at a single dose up to $3,000 \mu \mathrm{g}$ and in multiple doses up to $60 \mu \mathrm{g}$ without significant alterations of plasma lipids in healthy volunteers. 45

EDP-305 is also an FXR ligand. In animal models it improved liver fibrosis. ${ }^{46}$ It was evaluated for nonalcoholic steatohepatitis in a phase 2 a study (ARGON-1) by ENANTA Pharmaceuticals. EDP-305 efficiently reduced AST level after 12 weeks of therapy. Its adverse events were pruritus, nausea, vomiting, diarrhea, headache, and dizziness. One more trial with this agent is expected, a $2 b$ trial (Argon-2) for nonalcoholic steatohepatitis. ${ }^{47}$ Also a phase 2 study (INTREPID, NCT03394924) was completed in January 2020 on 68 participants with PBC. Results were improvements in ALT, AST, and markers of cholestasis. However, the primary
endpoint of at least $20 \%$ reduction in ALT was not met. ${ }^{48}$

Another interesting group of drugs are the PPAR agonists. PPARs have an important role in the metabolism of glucose and lipids and thus they have a great potential in therapy of MAFLD. PPARa appears to play a role in the development of MAFLD. PPARs also have a crucial role in beta-oxidation of lipids and play an important role in hepatic fibrosis. ${ }^{11}$ An agent from this group is fenofibrate. A study conducted in rats from 2012 showed amelioration of portal pressure and hepatic fibrosis. ${ }^{49} \mathrm{~A}$ pilot trail in 2010 (NCT00575042), including 20 PBC patients with an incomplete response to UDCA, published its results. Patients were treated with a combination of fenofibrate and UDCA. Results were significant improvements in liver biochemistry. The most prominent side effect was heartburn. ${ }^{50}$ Another study which included 90 patients with MAFLD published in 2015 did not show significant improvements in fibrosis. 51

Thiazolinediones, as PPARy ligands, are a group of insulinsensitizing medications, including rosiglitazone and pioglitazone. The FLIRT study from 2008 included 63 patients with MASH receiving rosiglitazone. Only half of the patients were responders and had improvements in liver biochemistry, but histopathological amelioration of fibrosis was absent. ${ }^{52}$ A FLIRT 2 trial (NCT00492700) was conducted in 2010, including 53 patients with MASH, among which 18 of them were treated with rosiglitazone. It improved liver biochemistry in the short term but in the long term it was not as efficient. ${ }^{53}$ Also, thiazolinediones have some side effects, such as weight gain, edema, bone loss, increased risk of bladder cancer, and cardiovascular complications, which limit their use. ${ }^{54}$ However, in a meta-analysis by Musso et al., 18 which included eight randomized clinical trials with 516 biopsyproven MASH patients, 24 months pioglitazone therapy was associated with reversal of advanced fibrosis, improved overall fibrosis stages, and resolution of $\mathrm{MASH}$, regardless of the presence of diabetes. ${ }^{55}$ The already mentioned PIVENS study (NCT0063622), which included 247 patients with MASH without diabetes, showed improvements in ALT and AST levels, and hepatic steatosis reduction. However, improvements in liver fibrosis were not detected. Data from a trial (NCT00994682) regarding pioglitazone were published in 2018. Participants were MASH patients, among which 52 had T2DM and 49 were prediabetic. Improvements were seen in histopathological findings of fibrosis and in insulin tissue sensitivity. ${ }^{56}$

A PPAR $\delta$ agonist seladelpar (MBX-8025) was tested in obese, dyslipidemic, and diabetic mouse model, where amelioration was confirmed by lipid levels, liver biochemistry results and hepatic cell balooning. ${ }^{57}$ Consequently, it is being evaluated for promising potential as a novel therapy for PSC in a phase 2 trial (NCT04024813) ${ }^{58}$ and for MASH (NCT03551522) and the results are expected in December 2020.59 Currently, there is also an ongoing phase 3 study (NCT03602560) including seladelpar (ENHANCE) in 240 participants with PBC. The results are expected in December 2021.60

Elafibranor (GFT-505) is a combined PPARa and PPARס agonist. A phase $2 b$ trial GOLDEN-505 (NCT01694849) was published in 2016 in patients with MASH, showing improvements in hepatic enzymes, levels of lipids and glucose. Elafibranor modestly resolved MASH histology, but it has not proven histological resolution of fibrosis. ${ }^{61}$ Currently, there is an ongoing clinical trial (RESOLVE-IT) for elafibranor in phase 3 investigation (NCT02704403). Final data are expected to become publicly available in 2021,62 when, based on confirmed efficacy and safety of elafibranor itself or in combinations with other developmental drugs, its possible role in the future treatment of MAFLD/MASH will be elucidated. 62,63 However, an interim analysis published in May 2020 has shown disappointing results, without reaching neither the primary nor secondary endpoint of the study. ${ }^{64}$

Saroglitazar is another representative from the PPAR dual ( $a$ and $y$ ) ligands group. It has been shown in preclinical studies that it targets all the segments of MASH. ${ }^{65}$ An analysis from 2019 has concluded that it ameliorates ALT levels and MAFLD.66 There are two active phase 2 clinical trials assessing saroglitazar in patients with MASH and assessing its safety in PBC. A phase 2 trial (NCT03061721, EVIDENCES IV) was completed in $106 \mathrm{MASH}$ patients, resulting in 
Rupcic Rubin V. et al: Antifibrotic agents: an update

amelioration of ALT levels, hepatic steatosis, dyslipidemia, and insulin resistance. 67 EVIDENCES VI (NCT03863574) will be finished in December 2020 and EVIDENCES VII in July 2021 (NCT03617263). ${ }^{68,69}$ Results of a phase 2 trial (EPICS, NCT03112681) assessing saroglitazar in PBC are expected to become publicly available in July $2020 .{ }^{70}$ However, the drug is already registered in India for diabetes treatment. ${ }^{4}$

There is one available pan-PPAR agonist, lanifibranor. Lanifibranor (IVA 337), a moderately potent and well-balanced pan-PPAR agonist ${ }^{71}$ is being evaluated as a potential drug candidate for MASH treatment in an ongoing $2 \mathrm{~b}$ trial (NCT03008070-NATIVE) by INVENTIVA. The results are expected to be published in 2020.72 Lanifibranor is also being investigated as a potential drug treatment of MAFLD and T2DM in another phase 2 trial (NCT03459079), and these results are expected in March 2021.73

Bezafibrate is a pan-PPAR agonist that was evaluated in patients with $\mathrm{PBC}$ who had an inadequate response to UDCA alone in a 24-month placebo-controlled phase 3 trial (NCT01654731-BEZURSO). Co-administration of bezafibrate to UDCA resulted in a complete biochemical response in $31 \%$ of patients, which was significantly higher than in the placebo and UDCA-treated group. Amelioration of pruritus, fatigue, and noninvasive measures of liver fibrosis were persistent, with observed enhancement of biochemical parameters. ${ }^{74}$

Telmisartan is a unique angiotensin receptor blocker (ARB) that modulates PPAR- $y$ activity and thereby increases sensitivity to insulin, which decreases hepatic fat accumulation. It also blocks the angiotensin II receptor, which inhibits hepatic stellate cell activation and suppresses hepatic fibrogenesis. ${ }^{75}$ One study (FANTASY) was conducted in a period from 2012 to 2014, comparing the effect of two ARBs (telmisartan and losartan) and aiming to support a theory that telmisartan might be more effective than losartan in MAFLD and in insulin resistance. Participants in this randomized controlled trial were patients with MAFLD, T2DM and hypertension treated with telmisartan and losartan. There was no remarkable enhancement in these two groups. Free fatty acids were decreased in the group telmisartan group. Also, one thing worth mentioning is that the liver-to-spleen ratio was better in the telmisartan group. ${ }^{75}$ Another prospective randomized controlled trial was conducted in 2016 on 35 MASH patients, in whom telmisartan plus life style modification were introduced, and $15 \mathrm{MASH}$ patients, in whom only life style modification was introduced. It revealed that telmisartan significantly improved the histology of MASH patients. One more trial was published in 2018 which included two groups of patients with MAFLD treated with placebo and telmisartan (dose of $20 \mathrm{mg}$ per day). Telmisartan decreased the level of IL-1 $\beta$ and Il-1 and thus had an antifibrotic effect. Neither telmisartan not placebo improved histopathology findings in MAFLD. ${ }^{76}$ Telmisartan side effects included sinus pain and congestion, back pain, diarrhea, sore throat, flu-like symptoms, upset stomach, muscle pain, headache, dizziness, fatigue, and nausea. ${ }^{77}$ Despite their potential benefit, ARBs are not used for non-cardiovascular indications because of dose-limiting hypotension. 78

The thyroid hormone receptor $\beta$ is a main liver receptor for thyroxine. There are two available selective ligands for MASH in this group, MGL-3196 (resmetirom), which initially targeted dyslipidemia, and VK2809. Results from a phase 2 trial (NCT02912260) including 348 patients with MASH that evaluated the use of resmetirom was published in 2019. Resmetirom-treated patients showed reduction in liver fat as assessed by MR imaging-proton density fat fraction. Reported side effects were mostly mild or moderate and balanced between groups, except for diarrhea and nausea. ${ }^{79}$ Resmetirom (MGL-3196) is also being investigated in an ongoing phase 3 trial (MAESTRO-NASH) in MASH and cirrhosis (NCT03900429) patients. The first results are expected to be published in March 2024.80 VK2809 showed improvements in liver lipids in a 3-month placebo-controlled phase 2 trial. ${ }^{4}$ VK2809 is currently in an ongoing phase $2 \mathrm{~b}$ trial for nonalcoholic steatohepatitis (NCT04173065-VOYAGE). The results are expected in November $2021 .{ }^{81}$ In 2019, a phase 2 study (NCT02927184) regarding VK2809 use in patients with MAFLD and hypercholesterolemia was completed. ${ }^{82}$ It showed amelioration in hepatic fat content in a 12-week period, with no serious side effects, as compared to placebo. Both ALT and AST levels were reduced. 83

ACC1 and ACC2 have a crucial role in fatty acid metabolism. Inhibition of ACC1 and ACC2 represents an interesting pharmacological approach for another group of agents targeting lipid metabolism through simultaneous inhibition of fatty acid synthesis and stimulation of fatty acid oxidation. An agent from this group is firsocostat. Recently, in a study on diet-induced rodent models of MAFLD, an improvement in liver steatosis was shown; however, a significant increase in plasma triglyceride levels was also observed. ${ }^{84}$ Therefore, based on the results of this study, the potential therapeutic utility of liver-directed ACC inhibition in the treatment of MAFLD remains to be further investigated. ${ }^{84}$ It is worth mentioning that this drug induced hypertriglyceridemia that has shown a good response to statin treatment. ${ }^{4}$ Also, a phase 2 trial (NCT02856555) evaluating safety and efficacy of GS-0976, an ACC inhibitor in liver, was recently completed and decrease in hepatic steatosis and selected markers of fibrosis, and liver biochemistry was confirmed. 85 However, reported side effects included pruritus, headache, diarrhea, and nausea. 29

Aramchol (arachidyl amido cholanoic acid) is a conjugate of fatty bile acids. It inhibits stearoyl coenzyme A desaturase 1 , which is crucial in lipogenesis. A phase 2 trial (ARREST) completed in 2018 showed improvements in hepatic fibrosis. ${ }^{86}$ Aramchol is currently in a phase 3 ongoing trial (ARMOR) for MASH (NCT04104321). ${ }^{87}$

There are also newly emerging indication opportunities for agents already used to treat diabetes, as numerous patients with MASH are diabetics as well. GLP-1 agonists exert various beneficial effects in T2DM, such as enhancement of glucose-dependent insulin secretion, acceleration of $\beta$-cell proliferation, inhibition of $\beta$-cell apoptosis, inhibition of motility and gastric emptying, and stimulation of the sensations of satiety and fullness by direct action on the central nervous system, with reduction in body weight. 88 Two agents that might be beneficial in MASH treatment are liraglutide and semaglutide. A phase 2 trial (NCT01237119, LEAN) investigating efficacy and safety of liraglutide, a long-acting GLP-1 analogue in nonalcoholic steatohepatitis patients, was published relatively recently. Amelioration of histopathology findings was confirmed; however, side effects including diarrhea, loss of appetite, and constipation were also observed. ${ }^{89}$ A phase 2 study (NCT 03987451) exploring efficacy and safety of semaglutide in MAFLD is ongoing and the results are expected to become publicly available in June 2021.90

A study was published in 2018 regarding a sodiumglucose cotransporter 2 inhibitor, canagliflozin. The results were amelioration of markers for hepatic fibrosis among included patients with T2DM and MASH. There were no serious side effects. As authors of the study stated, the limitation of this trial is a small number of participants. ${ }^{91} \mathrm{~A}$ phase 3 study (TCTR20190118008) evaluating canaglifozin in MASH was completed in March 2020 and the results are still expected. ${ }^{92}$

\section{Treatments targeting gut-liver axis}

The term "gut-liver axis" represents an anatomical and func- 
tional unit formed of the small intestine and liver. A number of signals, including hormones, chemokines and growth factors are released from the small intestine and enter the liver via the portal vein. Also, on the other hand, bile acids and other signals (IgA immunoglobulins) reach the small intestine via the bile. ${ }^{93}$ In a critical analysis of clinical trials in 2017, it was vividly described as "Whatever comes from the gut enters the liver and the portal circulation is the afferent and the biliary tree the efferent of the gut-liver-axis". ${ }^{94}$ In patients with liver fibrosis and cirrhosis, certain changes within the gut-liver axis have been demonstrated, including changes in the intestinal microbiota, as a potential target for future antifibrotic drugs. In a prospective study in 2017, it was demonstrated that MAFLD patients had a specific metagenomic-derived signature of fecal microbiota which enabled distinguishing patients with mild/moderate fibrosis from advanced fibrosis. ${ }^{95}$ In several experimental animal models the influence gut-derived signals, (e.g., intestinal mucus layer and the content of the intestinal microbiota on the fibrotic response in the liver) was determined. 96

Future perspectives of antifibrotic drugs include modulating the gut microbiome. Twenty-one randomized clinical trials on the effect of probiotics/synbiotics in MAFLD patients were evaluated in a recent meta-analysis. The authors reported reduction in ALT, improvement in liver stiffness measurement by elastography, and amelioration of steatosis as determined by ultrasound imaging, primarily as a probiotics/synbiotics effect on microbiome composition. 97 However, given the heterogeneity of the analyzed data and since neither of randomized clinical trials examined sequential histological findings, as determined by liver biopsy, the authors concluded that additional well-designed studies are needed to determine the true value of probiotics/synbiotics for treatment of liver fibrosis. It is worth mentioning that currently there is an ongoing randomized clinical trial (PROBILIVER TRIAL, NCT03467282) assessing 46 participants with MASH, receiving $1 \mathrm{~g}$ of probiotic mix in comparison to placebo. Results are expected in December 2021.98

Within the gut-liver axis field, promotion of useful gut signals could have promising results. In humans, FGF19 is a gut-derived hormone which regulates glucose homoeostasis and bile acid synthesis. Its beneficial metabolic effects were tested in a multicenter, randomized, double-blind, placebocontrolled, phase 2 clinical trial (NCT02443116) involving 82 biopsy-confirmed MASH patients with fibrosis stages 1-3. An engineered FGF19 analogue (NGM282, aldafermin) rapidly (after 12 weeks) and significantly diminished liver fat content, as measured by MR imaging. The vast majority of patients experienced at least one side effect, mostly the mildest (injection site reactions, diarrhea, abdominal pain, and nausea), while only a small percentage of patients experienced more severe side effects. ${ }^{99}$ Since the drug has an acceptable safety profile, further studies are approved. Currently, there is ongoing $2 \mathrm{~b}$ phase of multicenter, randomized, double-blind, placebo-controlled clinical trial (ALPINE 2/3, NCT03912532). The aim is to evaluate histologic response and also safety and tolerability of subcutaneously administered NGM282 for 24 weeks. The study included 152 histologically-confirmed MASH patients with fibrosis stage 2 or 3 . Still, there is no result data available and it is estimated to be completed in December 2020.100

\section{Treatments targeting fibrogenesis (myofibroblast ac- tivation and extracellular protein deposition)}

Studies on xenograft liver models determined 10 years ago that the key enzyme involved in extracellular collagen accumulation is lysyl oxidase-like homolog 2 (LOXL2). It significantly contributes to collagen stabilization catalyzing cross- linkage of collagen, and by such action has a profibrotic effect. Inhibition of LOXL2 using monoclonal antibodies had optimistic and promising preclinical results. ${ }^{101}$ Also, in animal models, irreversible inhibition of LOXL2 has resulted in decreased fibrosis in mice. ${ }^{102}$

However, the results from the $2 b$ phase of a clinical trials in MASH patients investigating the efficacy of simtuzumab as monoclonal antibody against LOXL2 were negative (NCT01672866, NCT01672879). Indeed, the study was terminated prematurely (after 96 weeks) for inefficiency of simtuzumab, which had not shown any beneficial effect when compared to placebo. ${ }^{103}$ Similar negative results were registered in a phase 2 study for PSC (NCT01672853) without significant reductions in fibrosis. ${ }^{104}$ Also, in a study including hepatitis $\mathrm{C}$ virus (commonly known as HCV) patients, human immunodeficiency virus (commonly known as HIV) patients, or patients with HCV-HIV co-infection and advanced liver disease, simtuzumab showed no improvement in liver fibrosis after 22 weeks. ${ }^{105}$

Inhibition of integrins, which are the receptors functioning in interactions between extracellular matrix and cells and also activators of pure profibrotic cytokine-transforming growth factor $\beta-1$, could have promising results in the treatment of hepatic fibrosis. Inhibitor of the av $\beta 6$ integrin (GSK3008348) was investigated in phase 1 clinical trial (NCT02612051) for the first time in humans for the treatment of idiopathic pulmonary fibrosis. The drug was well tolerated, with an acceptable safety profile; consequently, further studies are warranted. ${ }^{106}$

\section{Treatments targeting inflammation}

The boundaries between aforementioned pathways are arbitrary and, in vivo, these pathways overlap continuously. This is especially true for inflammatory pathomechanisms.

In liver fibrosis, one of the functionally important inflammatory but also fibrogenetic pathways for targeted therapeutic action is the expression of the carbohydrate molecule galectin 3 on inflammatory macrophages. Therefore, inhibition of this pathway by GR-MD-02 (galactoarabinorhamnogalaturonate, belapectin; Galectin Therapeutics) was tested in several rodent models, resulting in effective reduction of liver fibrosis. ${ }^{107}$ After 3 years of the first human phase 1 GT-020 study, the safety and efficacy of GRMD-02 has been demonstrated. ${ }^{108}$ Though, in two phase 2 clinical trials, the efficacy of GR-MD-02 in MASH patients did not produce the expected results. In a phase 2 clinical trial involving $30 \mathrm{MASH}$ patients with advanced fibrosis (NCT02421094), GR-MD-02 had no significant effect on non-invasive biomarkers of liver inflammation or fibrosis after 16 weeks, as measured by magnetic resonance elastography and shear-wave ultrasonic elastography, since histopathology monitoring was not planned for this study. ${ }^{109}$ In another phase $2 \mathrm{~b}$, randomized clinical trial of the safety and efficacy of GR-MD-02 involving 162 patients with MASH, cirrhosis, and portal hypertension (NCT02462967), GR-MD-02 showed no significant effect on liver fibrosis and liver-related outcomes. However, in a subgroup of patients without esophageal varices, GR-MD-02 has shown beneficial effects in alleviating portal hypertension and development of varices. ${ }^{110}$ According to a review from December 2019, a phase 3 clinical trial on GR-MD-02 in cirrhotic MASH patients began in the last quarter of 2019. ${ }^{11}$

The chemokine-receptor axis plays a pivotal role in liver inflammation pathways. In chronic liver disease, chemokines are released by Kupffer cells, stressed hepatocytes, endothelial and hepatic stellate cells. Liver inflammation is regulated by proinflammatory chemokines, promoting recruitment, migration and infiltration of inflammatory 
Rupcic Rubin V. et al: Antifibrotic agents: an update

cells (lymphocytes, monocytes, neutrophils) to the injury site. However, different chemokines and their receptors are characterized as the basis of various chronic liver diseases. Even more so, recent research has shown that certain chemokines and immune cells could have anti-inflammatory and antifibrotic impacts. ${ }^{111}$ One of the fundamental profibrotic pathways in chronic liver disease is the infiltration of inflammatory monocytes via the CC chemokine receptor (CCR)2. ${ }^{111}$ Accordingly, inhibition of CCR2 in an experimental murine model of MAFLD has confirmed reduction of hepatic inflammation and fibrosis. 112 Another chemokine receptor, CCR5, had also been determined to exert profibrogenic effects in murine models, predominantly via impact on hepatic stellate cell activation. ${ }^{113}$ An inhibitor of dual chemokine receptors type 2 and 5 (CCR2/CCR5) was developed by Allergan, based on previous successful preclinical research, and it was called cenicriviroc. In a 2-year randomized, double-blind, multinational phase $2 \mathrm{~b}$ clinical trial involving 289 subjects with noncirrhotic MASH and liver fibrosis (stages 1-3; CENTAUR (NCT02217475)), cenicriviroc has been evaluated as a promising antifibrotic therapeutic agent. ${ }^{114}$ Although the primary outcome of steatohepatitis alleviation has not been reached, the secondary endpoint of liver fibrosis improvement by $\geq 1$ stage was achieved after 1 year of therapy. Exploratory analyses after to 2 years of follow-up showed sustained treatment benefit, with greater effects in patients with advanced fibrosis. ${ }^{115}$ At a dose of $150 \mathrm{mg}$ daily, cenicriviroc showed a satisfactory safety profile in patients with mild-to-moderate hepatic impairment, with only a few drug-related side effects (i.e. fatigue with a frequency of $2.8 \%$, and diarrhea with a frequency of $2.1 \%) .115$ A phase 3 multicenter, randomized, doubleblind trial on efficacy and safety of cenicriviroc at a dose of $150 \mathrm{mg}$, known as the AURORA study (NCT03028740), is currently underway, with enrollment of 2,000 histologicallyproven MASH subjects with fibrosis stage F2 or F3. ${ }^{116}$

The third inflammatory target is the vascular adhesion protein-1 (VAP-1), also termed in the literature as amine oxidase copper-containing 3 . It is a membrane-bound amine oxidase, which has a dual effect in initiating lymphocyte migration to the site of hepatic injury and also promoting oxidative stress. ${ }^{117}$ In four murine models of hepatic injury, inhibition of VAP-1 resulted in reduced inflammatory cell recruitment to the liver and ameliorated liver fibrosis. ${ }^{117}$ Boehringer Ingelheim has been investigating BI 1467335 in a phase 2 a trial (NCT03166735), as a pharmacological inhibitor of VAP-1 in 150 MASH patients. ${ }^{118}$ However, the company decided to discontinue the study for MASH due to risk of drug interactions. ${ }^{119}$

\section{Conclusions}

Liver fibrosis remains without approved pharmacotherapy. The treatment of the underlying disease, if possible, is the sole approach, with liver transplantation being the ultimate therapeutic option. For cases of MAFLD, representing the leading cause of liver fibrosis in the world, only a few specific therapeutic recommendations are available. ${ }^{118}$ Since fibrosis, as a consequence of chronic liver injury, is considered a key factor in the prognosis and overall mortality, its resolution is the main goal in new antifibrotic therapeutic approaches. Basic research and clinical trials have reinforced the complexity of underlying signaling pathways in liver fibrosis development. In this context, novel antifibrotic drugs are targeting cell death, abnormal liver metabolism, the gut-liver axis, myofibroblast activation, extracellular protein deposition, and inflammation. Promising new antifibrotic compounds currently in phase 3 clinical trials are obeticholic acid (FXR agonism) in REVERSE trials, elafibranor (dual
PPAR a/ $\delta$ agonist) in the RESOLVE-IT study, and cenicriviroc (CCR2/CCR5 inhibitor) in the AURORA study. A key issue in the future will be potential combination therapy with a synergistic effect, which could target multiple underlying pathophysiological mechanisms of fibrosis. The modest effect sizes of different antifibrotic drugs will likely lead to pursuit of drug combinations personalized to each stage of the MAFLD disease spectrum.

\section{Funding}

The study was funded by grant from Croatian Ministry of Science and Education dedicated to multi-year institutional funding of scientific activity at the J.J. Strossmayer University of Osijek, Osijek, Croatia-grant number: IP6-MEFOS-2019 (to R.S.).

\section{Conflict of interest}

The authors have no conflict of interests related to this publication.

\section{Author contributions}

Conceived of and designed the article, and critically revised the manuscript (RS, MS), performed critical revision of the manuscript for important intellectual content, obtained funding, and provided administrative, technical and material support (RS), performed literature searches and wrote the manuscript (VRR, KB), updated the text of the manuscript (MS, RS), performed literature searches and table and diagram drawing (JR), critical revision of the manuscript for important intellectual content (AT).

\section{References}

[1] Tacke F, Weiskirchen R. An update on the recent advances in antifibrotic therapy. Expert Rev Gastroenterol Hepatol 2018;12:1143-1152. doi:10.1 080/17474124.2018.1530110.

[2] Younossi ZM, Stepanova M, Younossi Y, Golabi P, Mishra A, Rafiq N, et al. Epidemiology of chronic liver diseases in the USA in the past three decades. Gut 2020;69:564-568. doi:10.1136/gutjnl-2019-318813.

[3] Eslam M, Newsome PN, Sarin SK, Anstee QM, Targher G, Romero-Gomez $M$, et al. A new definition for metabolic dysfunction-associated fatty liver disease: An international expert consensus statement. J Hepatol 2020; 73:202-209. doi:10.1016/j.jhep.2020.03.039.

[4] Lemoinne S, Friedman SL. New and emerging anti-fibrotic therapeutics entering or already in clinical trials in chronic liver diseases. Curr Opin Pharmacol 2019;49:60-70. doi:10.1016/j.coph.2019.09.006.

[5] Dulai PS, Singh S, Patel J, Soni M, Prokop LJ, Younossi Z, et al. Increased risk of mortality by fibrosis stage in nonalcoholic fatty liver disease: Systematic review and meta-analysis. Hepatology 2017;65:1557-1565. doi:10.1002/hep.29085.

[6] Pan JJ, Bao F, Du E, Skillin C, Frenette CT, Waalen J, et al. Morphometry confirms fibrosis regression from sustained virologic response to directacting antivirals for hepatitis C. Hepatol Commun. 2018;2:1320-1330. doi:10.1002/hep4.1228.

[7] D'Ambrosio R, Aghemo A, Rumi MG, Ronchi G, Donato MF, Paradis V, et al. A morphometric and immunohistochemical study to assess the benefit of a sustained virological response in hepatitis $C$ virus patients with cirrhosis. Hepatology 2012;56:532-543. doi:10.1002/hep.25606.

[8] Zoubek ME, Trautwein C, Strnad P. Reversal of liver fibrosis: From fiction to reality. Best Pract Res Clin Gastroenterol 2017;31:129-141. doi:10.1016/j. bpg.2017.04.005.

[9] Lee Y, Doumouras AG, Yu J, Brar K, Banfield L, Gmora S, et al. Complete resolution of nonalcoholic fatty liver disease after bariatric surgery: A systematic review and meta-analysis. Clin Gastroenterol Hepatol gery: A systematic review and meta-analysis. Clin Gastroent

[10] Balmaceda JB, Aepfelbacher J, Belliveau O, Chaudhury CS, Chairez C, McLaughlin M, et al. Long-term changes in hepatic fibrosis following hepatitis $C$ viral clearance in patients with and without HIV. Antivir Ther 2019;24:451-457. doi:10.3851/IMP3327.

[11] Santoro R, Mangia A. Progress in promising anti-fibrotic therapies, Expert Rev Gastroenterol Hepatol 2019;13:1145-1152. doi:10.1080/17474124.2 
019.1688659

[12] Luedde T, Kaplowitz N, Schwabe RF. Cell death and cell death responses in liver disease: mechanisms and clinical relevance. Gastroenterology 2014;147:765-783.e4. doi:10.1053/j.gastro.2014.07.018.

[13] Eguchi A, Wree A, Feldstein AE. Biomarkers of liver cell death. J Hepatol 2014;60:1063-1074. doi:10.1016/j.jhep.2013.12.026.

[14] Tsuchida T, Friedman SL. Mechanisms of hepatic stellate cell activation. Nat Rev Gastroenterol Hepatol 2017;14:397-411. doi:10.1038/nrgastro.2017.38.

[15] Nagashimada M, Ota T. Role of vitamin E in nonalcoholic fatty liver disease. IUBMB Life 2019;71:516-522. doi:10.1002/iub.1991.

[16] Perumpail B], Li AA, John N, Sallam S, Shah ND, Kwong W, et al. The role of vitamin $E$ in the treatment of NAFLD. Diseases 2018;6:86. doi:10.3390/ diseases 6040086

[17] El Hadi H, Vettor R, Rossato M. Vitamin E as a treatment for nonalcoholic fatty liver disease: Reality or myth? Antioxidants (Basel) 2018;7:12. doi:10.3390/antiox7010012.

[18] Sanyal AJ, Chalasani N, Kowdley KV, McCullough A, Diehl AM, Bass NM, et al. Pioglitazone, vitamin $\mathrm{E}$, or placebo for nonalcoholic steatohepatitis. N Engl J Med 2010;362:1675-1685. doi:10.1056/NEJMoa0907929.

[19] Xu R, Tao A, Zhang S, Deng Y, Chen G. Association between vitamin E and non-alcoholic steatohepatitis: a meta-analysis. Int J Clin Exp Med 2015;8:3924-3934

[20] Barreyro FJ, Holod S, Finocchietto PV, Camino AM, Aquino JB, Avagnina A, et al. The pan-caspase inhibitor Emricasan (IDN-6556) decreases liver injury and fibrosis in a murine model of non-alcoholic steatohepatitis. Liver Int 2015;35:953-966. doi:10.1111/liv.12570.

[21] Gracia-Sancho J, Manicardi N, Ortega-Ribera M, Maeso-Díaz R, GuixéMuntet S, Fernández-Iglesias A, et al. Emricasan ameliorates portal hypertension and liver fibrosis in cirrhotic rats through a hepatocyte-mediated paracrine mechanism. Hepatol Commun 2019;3:987-1000. doi:10.1002/ hep4.1360.

[22] Harrison SA, Goodman Z, Jabbar A, Vemulapalli R, Younes ZH, Freilich $\mathrm{B}$, et al. A randomized, placebo-controlled trial of emricasan in patients with NASH and F1-F3 fibrosis. J Hepatol 2020;72:816-827. doi:10.1016/j. jhep.2019.11.024.

[23] Yamamoto E, Dong YF, Kataoka K, Yamashita T, Tokutomi Y, Matsuba S, et al. Olmesartan prevents cardiovascular injury and hepatic steatosis in et al. Olmesartan prevents cardiovascular injury and hepatic steatosis in
obesity and diabetes, accompanied by apoptosis signal regulating kinase-1 inhibition. Hypertension 2008;52:573-580. doi:10.1161/HYPERTENSIONAHA.108.112292.

[24] Budas G, Karnik S, Jonnson T, Shafizadeh T, Watkins S, Breckenridge $D_{\text {, }}$ et al. Reduction of liver steatosis and fibrosis with an Ask1 inhibitor in a murine model of Nash is accompanied by improvements in cholesterol, bile acid and lipid metabolism. J Hepatol 2016;64:S170. doi:10.1016/S01688278(16)01686-X

[25] Loomba R, Lawitz E, Mantry PS, Jayakumar S, Caldwell SH, Arnold H, et al. The ASK1 inhibitor selonsertib in patients with nonalcoholic steatohepatitis: A randomized, phase 2 trial. Hepatology 2018;67:549-559. doi: 10.1002 /hep.29514

[26] Gilead Sciences Inc. Gilead announces topline data from phase 3 STELLAR-3 study of selonsertib in bridging fibrosis (F3) due to nonalcoholic steatohepatitis (NASH). Available from: https://www.gilead.com/newsand-press/press-room/press-releases/2019/4/gilead-announces-toplinedata-from-phase-3-stellar3-study-of-selonsertib-in-bridging-fibrosis-f3data-from-phase-3-stellar3-study-of-selons

[27] Gilead Sciences Inc. Gilead announces topline data from phase 3 STELLAR-4 study of selonsertib in compensated cirrhosis (F4) due to nonalcoholic steatohepatitis (NASH). Available from: https://www.gilead.com/ news-and-press/press-room/press-releases/2019/2/gilead-announces topline-data-from-phase-3-stellar4-study-of-selonsertib-in-compensatedcirrhosis-f4-due-to-nonalcoholic-steatohepatitis-nash.

[28] Study to evaluate the safety and efficacy of Selonsertib, Firsocostat, Cilofexor, and com-binations in participants with bridging fibrosis or compensated cirrhosis due to nonalco-holic steatohepatitis (NASH) (ATLAS), NCT03449446. Available from: https://clinicaltrials.gov/ct2/show/results/ NCT03449446.

[29] Gilead Sciences Inc. Gilead announces topline results from phase 2 ATLAS study in pa-tients with bridging fibrosis (F3) and compensated cirrhosis (F4) due to nonalcoholic stea-tohepatitis (NASH). Available from: https:// www.gilead.com/news-and-press/press-room/press-releases/2019/12/ gilead-announces-topline-results-from-phase-2-atlas-study-in-patientswith-bridging-fibrosis-f3-and-compensated-cirrhosis-f4-due-to-nonalcoholic-s.

[30] Reimer KC, Wree A, Roderburg C, Tacke F. New drugs for NAFLD: lessons from basic models to the clinic. Hepatol Int 2020;14:8-23. doi:10.1007/ s12072-019-10001-4

[31] Tang N, Zhang Y, Liang Q, Liu Z, Shi Y. The role of ursodeoxycholic acid on cholestatic hepatic fibrosis in infant rats. Mol Med Rep 2018;17:38373844. doi: $10.3892 / \mathrm{mmr}$.2017.8284.

[32] Parikh P, Ingle M, Patel J, Bhate P, Pandey V, Sawant P. An open-label randomized control study to compare the efficacy of vitamin e versus ursodeoxycholic acid in nondiabetic and noncirrhotic Indian NAFLD patients. Saudi oxycholic acid in nondiabetic and noncirrhotic Indian NAFLD patients.

[33] Kolarić TO, Ninčević V, Smolić R, Smolić M, Wu GY. Mechanisms of hepatic cholestatic drug injury. J Clin Transl Hepatol 2019; 7:86-92. doi:10.14218/ JCTH.2018.00042.

[34] Wang H, Ge C, Zhou J, Guo Y, Cui S, Huang N, et al. Noncanonical farnesoid $X$ receptor signaling inhibits apoptosis and impedes liver fibrosis. EBioMedicine 2018;37:322-333. doi:10.1016/j.ebiom.2018.10.028.

[35] Cipriani S, Mencarelli A, Palladino G, Fiorucci S. FXR activation reverses in- sulin resistance and lipid abnormalities and protects against liver steatosis in Zucker (fa/fa) obese rats. J Lipid Res 2010;51:771-784. doi:10.1194/ jlr.M001602.

[36] Verbeke L, Mannaerts I, Schierwagen R, Govaere O, Klein S, Vander Elst I, et al. FXR agonist obeticholic acid reduces hepatic inflammation and fibrosis in a rat model of toxic cirrhosis. Sci Rep 2016;6:33453. doi:10.1038/ srep33453.

[37] Younossi Z, Ratziu V, Loomba R, Rinella M, Anstee QM, Zaru L, et al. OTU-14 Positive results from REGENERATE: a phase 3 international, randomized, placebo-controlled study evaluating obeticholic acid treatment for NASH. Gut 2019;68:A108. doi:10.1136/gutjnl-2019-BSGAbstracts.205.

[38] Neuschwander-Tetri BA, Loomba R, Sanyal AJ, Lavine JE, Van Natta ML, Abdelmalek MF, et al. Farnesoid $X$ nuclear receptor ligand obeticholic acid for non-cirrhotic, non-alcoholic steatohepatitis (FLINT): a multicentre, randomised, placebo-controlled trial. Lancet 2015;385:956-965. doi:10.1016/S0140-6736(14)61933-4.

[39] Aschenbrenner DS. Excessive dosing of obeticholic acid may increase risk of liver damage. Am J Nurs 2018;118:46. doi:10.1097/01.NAJ.0000 530245.53335.c8

[40] Trauner M, Gulamhusein A, Hameed B, Caldwell S, Shiffman ML, Landis C, et al. The nonsteroidal farnesoid X receptor agonist cilofexor (GS-9674) improves markers of cholestasis and liver injury in patients with primary sclerosing cholangitis. Hepatology 2019;70:788-801. doi:10.1002/ hep.30509.

[41] Safety, tolerability, and efficacy of Cilofexor in non-cirrhotic adults with primary scleros-ing cholangitis (PRIMIS), NCT03890120. Available from: https://clinicaltrials.gov/ct2/show/NCT03890120.

[42] Hernandez ED, Zheng L, Kim Y, Fang B, Liu B, Valdez RA, et al. Tropifexor-mediated abrogation of steatohepatitis and fibrosis is associated with the antioxidative gene expression profile in rodents. Hepatol Commun 2019;3:1085-1097. doi:10.1002/hep4.1368.

[43] Study of safety and efficacy of Tropifexor (LJN452) in patients with nonalcoholic steato-hepatitis (NASH) (FLIGHT-FXR), NCT02855164. Available from: https://clinicaltrials.gov/ct2/show/NCT02855164.

[44] Novartis. Novartis data show tropifexor (LJN452) significantly improves several key biomarkers of NASH in patients with moderate to severe fibrosis. Available from: https://www novartis.com/news/media-releases/ novartis-data-show-tropifexor-ljn452-significantly-improves-several-keybiomarkers-nash-patients-moderate-severe-fibrosis.

[45] Badman MK, Chen J, Desai S, Vaidya S, Neelakantham S, Zhang J, et al. Safety, tolerability, pharmacokinetics, and pharmacodynamics of the nove non-bile acid FXR agonist tropifexor (LJN452) in healthy volunteers. Clin Pharmacol Drug Dev 2020;9:395-410. doi:10.1002/cpdd.762.

[46] Erstad DJ, Farrar CT, Ghoshal S, Masia R, Ferreira DS, Chen YI, et al. Molecular magnetic resonance imaging accurately measures the antifibrotic effect of EDP-305, a novel farnesoid X receptor agonist. Hepatol Commun 2018;2:821-835. doi:10.1002/hep4.1193.

[47] Enanta announces positive results of argon-1 study of its lead FXR agonist, EDP-305, for the treatment of NASH. Available from: https://www.enanta. com/investors/news-releases/press-release/2019/Enanta-Announces-Positive-Results-of-ARGON-1-Study-of-its-lead-FXR-Agonist-EDP-305-for-theTreatment-of-NASH/default.aspx.

[48] Enanta announces results of INTREPID study of EDP-305 for the treatment of primary biliary cholangitis. Available from: https://www.enanta.com/ investors/news-releases/press-release/2020/Enanta-Announces-Resultsof-INTREPID-Study-of-EDP-305-for-the-Treatment-of-Primary-BiliaryCholangitis/default.aspx.

[49] Rodríguez-Vilarrupla A, Laviña B, García-Calderó H, Russo L, Rosado $\mathrm{E}$, Roglans N, et al. PPARa activation improves endothelial dysfunction and reduces fibrosis and portal pressure in cirrhotic rats. J Hepatol 2012;56:1033-1039. doi:10.1016/j.jhep.2011.12.008.

[50] Levy C, Peter JA, Nelson DR, Keach J, Petz J, Cabrera R, et al. Pilot study: fenofibrate for patients with primary biliary cirrhosis and an incomplete response to ursodeoxycholic acid. Aliment Pharmacol Ther 2011;33:235242. doi:10.1111/j.1365-2036.2010.04512.x

[51] El-Haggar SM, Mostafa TM. Comparative clinical study between the effect of fenofibrate alone and its combination with pentoxifylline on biochemical parameters and liver stiffness in patients with non-alcoholic fatty liver disease. Hepatol Int 2015;9:471-479. doi:10.1007/s12072-015-9633-1.

[52] Ratziu V, Giral P, Jacqueminet S, Charlotte F, Hartemann-Heurtier A, Serfaty $L$, et al. Rosiglitazone for nonalcoholic steatohepatitis: one-year results of the randomized placebo-controlled Fatty Liver Improvement with Rosiglitazone Therapy (FLIRT) Trial. Gastroenterology 2008;135:100-110. doi:10.1053/j.gastro.2008.03.078.

[53] Ratziu V, Charlotte F, Bernhardt C, Giral P, Halbron M, Lenaour G, et al. Long-term efficacy of rosiglitazone in nonalcoholic steatohepatitis: results of the fatty liver improvement by rosiglitazone therapy (FLIRT 2) extension trial. Hepatology 2010;51:445-453. doi:10.1002/hep.23270.

[54] Liss KH, Finck BN. PPARs and nonalcoholic fatty liver disease. Biochimie 2017;136:65-74. doi:10.1016/j.biochi.2016.11.009.

[55] Musso G, Cassader M, Paschetta E, Gambino R. Thiazolidinediones and advanced liver fibrosis in nonalcoholic steatohepatitis: A meta-analysis. JAMA Intern Med 2017;177:633-640. doi:10.1001/jamainternmed.2016.9607.

[56] Bril F, Kalavalapalli S, Clark VC, Lomonaco R, Soldevila-Pico C, Liu IC, et al. Response to pioglitazone in patients with nonalcoholic steatohepatitis with vs without type 2 diabetes. Clin Gastroenterol Hepatol 2018;16:558-566. e2. doi:10.1016/j.cgh.2017.12.001.

[57] Haczeyni F, Wang H, Barn V, Mridha AR, Yeh MM, Haigh WG, et al. The selective peroxisome proliferator-activated receptor-delta agonist seladelpar reverses nonalcoholic steatohepatitis pathology by abrogating lipotoxicity in diabetic obese mice. Hepatol Commun 2017;1:663-674. doi:10.1002/ 
hep4.1072.

[58] A study to evaluate the safety, and tolerability, and efficacy of Seladelpar in patients with PSC, NCT04024813. Available from: https://clinicaltrials. gov/ct2/show/NCT04024813/.

[59] A study to evaluate Seladelpar in subjects with nonalcoholic steatohepatitis (NASH), NCT03551522. Available from: https://clinicaltrials.gov/ct2/ show/NCT03551522/.

[60] ENHANCE: Seladelpar in subjects with primary biliary cholangitis (PBC) and an inade-quate response to or an intolerance to ursodeoxycholic acid (UDCA), NCT03602560. Available from: https://clinicaltrials.gov/ct2/show/ NCT03602560/.

[61] Ratziu V, Harrison SA, Francque S, Bedossa P, Lehert P, Serfaty L, et al. Elafibranor, an agonist of the peroxisome proliferator-activated receptor- 0 and $-\delta$, induces resolution of nonalcoholic steatohepatitis without fibrosis worsening. Gastroenterology 2016;150:1147-1159.e5. doi:10.1053/j. gastro.2016.01.038

[62] Phase 3 study to evaluate the efficacy and safety of Elafibranor versus placebo in patients with nonalcoholic steatohepatitis (NASH) (RESOLVEIT), NCT02704403. Available from: https://clinicaltrials.gov/ct2/show/ NCT02704403/.

[63] Westerouen Van Meeteren MJ, Drenth JPH, Tjwa ETTL. Elafibranor: a potential drug for the treatment of nonalcoholic steatohepatitis (NASH). Ex pert Opin Investig Drugs 2020;29:117-123. doi:10.1080/13543784.2020 .1668375 .

[64] GENFIT: Announces results from interim analysis of RESOLVE-IT phase 3 trial of Elafi-branor in adults with NASH and fibrosis. Available from: https://www.globenewswire.com/news-release/2020/05/11/2031418/0/en/ GENFIT-Announces-Results-from-Interim-Analysis-of-RESOLVE-IT-PhaseGENFIT-Announces-Results-from-Interim-Analysis-of-RESOLV-
3-Trial-of-Elafibranor-in-Adults-with-NASH-and-Fibrosis.html.

[65] Zydus announces positive results from EVIDENCES IV phase 2 clinical trial of saroglita-zar magnesium in NAFLD and NASH. Available from: https:// www.prnewswire.com/news-releases/zydus-announces-positive-resultsfrom-evidences-iv-phase-2-clinical-trial-of-saroglitazar-magnesium-innafld-and-nash-300930487.html.

[66] Kaul U, Parmar D, Manjunath K, Shah M, Parmar K, Patil KP, et al. New dual peroxisome proliferator activated receptor agonist-Saroglitazar in diabetic dyslipidemia and non-alcoholic fatty liver disease: integrated analysis of dyslipidemia and non-alcoholic fatty liver disease: integrated analysis of the real world evidence

[67] Saroglitazar magnesium in patients with nonalcoholic fatty liver disease and/or nonalco-holic steatohepatitis (EVIDENCES IV), NCT03061721. Available from: https://clinicaltrials.gov/ct2/show/NCT03061721.

[68] Saroglitazar magnesium in the treatment of non-alcoholic steatohepatitis (EVIDENCES VI), NCT03863574. Available from: https://clinicaltrials.gov/ ct2/show/NCT03863574.

[69] Saroglitazar magnesium $4 \mathrm{mg}$ in the treatment of NAFLD in women with PCOS (EVI-DENCES VII), NCT03617263. Available from: https://clinicaltrials.gov/ct2/show/NCT03617263/.

[70] Study to evaluate safety, tolerability and efficacy of saroglitazar Mg in patients with pri-mary biliary cholangitis (EPICS), NCT03112681. Available from: https://clinicaltrials.gov/ct2/show/NCT03112681/.

[71] Boubia B, Poupardin O, Barth M, Binet J, Peralba P, Mounier L, et al. Design, synthesis, and evaluation of a novel series of indole sulfonamide peroxisome proliferator activated receptor (PPAR) $a / Y / \delta$ triple activators: Discovery of lanifibranor, a new antifibrotic clinical candidate. J Med Chem 2018;61:2246-2265. doi:10.1021/acs.jmedchem.7b01285.

[72] Phase 2b study in NASH to assess IVA337 (NATIVE), NCT03008070. Available from: https://clinicaltrials.gov/ct2/show/NCT03008070.

[73] Lanifibranor in patients with type 2 diabetes \& nonalcoholic fatty liver disease, NCT03459079. Available from: https://clinicaltrials.gov/ct2/show/ NCT03459079.

[74] Corpechot C, Chazouillères O, Rousseau A, Le Gruyer A, Habersetzer F, Mathurin $\mathrm{P}$, et al. A Placebo-controlled trial of bezafibrate in primary biliary cholangitis. N Engl J Med 2018;378:2171-2181. doi:10.1056/NEJMoa171 4519.

[75] Alam S, Kabir J, Mustafa G, Gupta U, Hasan SK, Alam AK. Effect of telmisartan on histological activity and fibrosis of non-alcoholic steatohepatitis: A 1-year randomized control trial. Saudi J Gastroenterol 2016;22:6976. doi:10.4103/1319-3767.173762.

[76] Al-Nimer MSM, Esmail VAW, Mohammad O. Telmisartan improves the metabolic, hematological and inflammasome indices in non-alcoholic fatty live infiltration: A pilot open-label placebo-controlled study. Electron J Gen Med 2019;16:em142. doi:10.29333/ejgm/104568.

[77] Telmisartan (Oral Route). Available from: https://www.mayoclinic.org/drugssupplements/telmisartan-oral-route/side-effects/drg-20067196? $p=1$.

[78] Golder MR, Liu J, Andersen JN, Shipitsin MV, Vohidov F, Nguyen HV, et al. Reduction of liver fibrosis by rationally designed macromolecular telmisartan prodrugs. Nat Biomed Eng 2018;2:822-830. doi:10.1038/s41551018-0279- $x$

[79] Harrison SA, Bashir MR, Guy CD, Zhou R, Moylan CA, Frias JP, et al. Resmetirom (MGL-3196) for the treatment of non-alcoholic steatohepatitis: a multicentre, randomised, double-blind, placebo-controlled, phase 2 trial. Lancet 2019;394:2012-2024. doi:10.1016/S0140-6736(19)32517-6.

[80] A phase 3 study to evaluate the efficacy and safety of MGL-3196 (Resmetirom) in pa-tients with NASH and fibrosis (MAESTRO-NASH), NCT03900429. Available from: https://clinicaltrials.gov/ct2/show/NCT03900429.

[81] A study to assess the efficacy and safety of VK2809 for 52 weeks in subjects with biopsy proven NASH (VOYAGE), NCT04173065. Available from https://clinicaltrials.gov/ct2/show/NCT04173065/.

[82] Safety and tolerability of VK2809 in patients with primary hypercholesterolemia and non-alcoholic fatty liver disease, NCT02927184. Available from: https://clinicaltrials.gov/ct2/show/NCT02927184.

[83] Therapeutics V. Viking therapeutics presents results from phase 2 study of VK2809 in patients with non-alcoholic fatty liver disease (NAFLD) and elevated LDL-cholesterol in oral late-breaker presentation at the liver meeting ${ }^{\circledR}$ 2018. Available from: https://www.prnewswire.com/news-releases/vikingtherapeutics-presents-results-from-phase-2-study-of-vk2809-in-patientswith-non-alcoholic-fatty-liver-disease-nafld-and-elevated-Idl-cholesterolin-oral-late-breaker-presentation-at-the-liver-meeting-2018-300748744. html.

[84] Goedeke L, Bates J, Vatner DF, Perry RJ, Wang T, Ramirez R, et al. AcetylCoA carboxylase inhibition reverses NAFLD and hepatic insulin resistance but promotes hypertriglyceridemia in rodents. Hepatology 2018;68:21972211. doi:10.1002/hep.30097.

[85] Lawitz EJ, Coste A, Poordad F, Alkhouri N, Loo N, McColgan B], et al. Acetyl-CoA carboxylase inhibitor GS-0976 for 12 weeks reduces hepatic de novo lipogenesis and steatosis in patients with nonalcoholic steatohepatitis. Clin Gastroenterol Hepatol 2018;16:1983-1991.e3. doi:10.1016/j. cgh.2018.04.042.

[86] Ratziu V, de Guevara L, Safadi R, Poordad F, Fuster F, Flores-Figueroa J, et al. One - year results of the Global Phase $2 b$ randomized placebo - controlled ARREST Trial of Aramchol, a Stearoyl CoA Desaturase modulator in NASH patients. The AASLD Liver Meeting. San Francisco 2018.

[87] A phase $3 / 4$ clinical study to evaluate the efficacy and safety of Aramchol versus placebo in subjects with NASH (ARMOR) (ARMOR), NCT04104321. Available from: https://clinicaltrials.gov/ct2/show/NCT04104321.

[88] Ninčević V, Omanović Kolarić T, Roguljić H, Kizivat T, Smolić M, Bilić Ćurčić I. Renal benefits of SGLT 2 inhibitors and GLP-1 receptor agonists: Evidence supporting a paradigm shift in the medical management of type 2 dence supporting a paradigm shift in the medical management of
diabetes. Int J Mol Sci 2019;20:5831. doi:10.3390/ijms20235831.

[89] Armstrong MJ, Gaunt P, Aithal GP, Barton D, Hull D, Parker R, et al. Liraglutide safety and efficacy in patients with non-alcoholic steatohepatitis (LEAN): a multicentre, double-blind, randomised, placebo-controlled phase 2 study. Lancet 2016;387:679-690. doi:10.1016/S0140-6736(15)00803$X$.

[90] A research study on how Semaglutide works in people with fatty liver disease and liver damage, NCT03987451. Available from: https://clinicaltrials.gov/ct2/show/NCT03987451.

[91] Seko Y, Nishikawa T, Umemura A, Yamaguchi K, Moriguchi M, Yasui K, et al. Efficacy and safety of canagliflozin in type 2 diabetes mellitus patients with biopsy-proven nonalcoholic steatohepatitis classified as stage 1-3 fibrosis. Diabetes Metab Syndr Obes 2018;11:835-843. doi:10.2147/DMSO. S184767.

[92] Effect of Canagliflozin on liversteatosis in obese non diabetic patients with nonalcoholic fatty liver disease: A double blind randomized controlled trial (CANA-NAFLD), TCTR20190118008. Available from: http://www.clinicaltrials. in th/index php?tp $=$ regtrials\&menu $=$ trialsearch\&smenu $=$ fulltext\&task $=$ in.th/index.php?tp $=$ regtrials\&

[93] Delacroix DL, Hodgson HJ, McPherson A, Dive C, Vaerman JP. Selective transport of polymeric immunoglobulin A in bile. Quantitative relationships of monomeric and polymeric immunoglobulin $A$, immunoglobulin $M$, and other proteins in serum, bile, and saliva. J Clin Invest 1982;70:230-241. doi:10.1172/jci110610.

[94] Wiest R, Albillos A, Trauner M, Bajaj JS, Jalan R. Targeting the gut-liver axis in liver disease. J Hepatol 2017;67:1084-1103. doi:10.1016/j. jhep.2017.05.007.

[95] Loomba R, Seguritan V, Li W, Long T, Klitgord N, Bhatt A, et al. Gut microbiome-based metagenomic signature for non-invasive detection of advanced fibrosis in human nonalcoholic fatty liver disease. Cell Metab 2017;25:1054-1062.e5. doi:10.1016/j.cmet.2017.04.001.

[96] De Minicis S, Rychlicki C, Agostinelli L, Saccomanno S, Candelaresi C, Trozzi $L$, et al. Dysbiosis contributes to fibrogenesis in the course of chronic liver injury in mice. Hepatology 2014;59:1738-1749. doi:10.1002/hep.26695.

[97] Sharpton SR, Maraj B, Harding-Theobald E, Vittinghoff E, Terrault NA Gut microbiome-targeted therapies in nonalcoholic fatty liver disease: a systematic review, meta-analysis, and meta-regression. Am J Clin Nutr 2019;110:139-149. doi:10.1093/ajcn/nqz042.

[98] Silva-Sperb AS, Moraes HA, de Moura BC, Alves BC, Bruch-Bertani JP, Azevedo VZ, et al. Effect of probiotic supplementation in nonalcoholic steatohepatitis patients: PROBILIVER TRIAL protocol. Trials 2019;20:580. doi:10.1186/s13063-019-3679-7

[99] Harrison SA, Rinella ME, Abdelmalek MF, Trotter JF, Paredes AH, Arnold $\mathrm{HL}$, et al. NGM282 for treatment of non-alcoholic steatohepatitis: a multicentre, randomised, double-blind, placebo-controlled, phase 2 trial. Lancet 2018;391:1174-1185. doi:10.1016/S0140-6736(18)30474-4.

[100] Evaluation of efficacy, safety and tolerability of Aldafermin in a phase $2 \mathrm{~b}$, randomized, double-blind, placebo-controlled, multi-center study in subjects with nonalcoholic steato-hepatitis and stage $2 / 3$ fibrosis (ALPINE 2/3), NCT03912532. Available from: https://clinicaltrials.gov/ct2/show/ NCT03912532.

[101] Barry-Hamilton V, Spangler R, Marshall D, McCauley S, Rodriguez HM, Oyasu M, et al. Allosteric inhibition of lysyl oxidase-like-2 impedes the development of a pathologic microenvironment. Nat Med 2010;16:10091017. doi: $10.1038 / \mathrm{nm} .2208$.

[102] Liu SB, Ikenaga N, Peng ZW, Sverdlov DY, Greenstein A, Smith V, et al. Lysyl oxidase activity contributes to collagen stabilization during liver fibrosis progression and limits spontaneous fibrosis reversal in mice. FASEB J 2016;30:1599-1609. doi:10.1096/fj.14-268425

[103] Sanyal AJ, Harrison SA, Ratziu V, Abdelmalek MF, Diehl AM, Caldwell S, et al. The natural history of advanced fibrosis due to nonalcoholic steatohepatitis: Data from the simtuzumab trials. Hepatology 2019;70:1913-1927. doi:10.1002/hep.30664. 
[104] Muir AJ, Levy C, Janssen HLA, Montano-Loza AJ, Shiffman ML, Caldwell $\mathrm{S}$, et al. Simtuzumab for primary sclerosing cholangitis: Phase 2 study results with insights on the natural history of the disease. Hepatology 2019;69:684-698. doi:10.1002/hep.30237.

[105] Meissner EG, McLaughlin M, Matthews L, Gharib AM, Wood B], Levy E, et al. Simtuzumab treatment of advanced liver fibrosis in HIV and HCV infected adults: results of a 6-month open-label safety trial. Liver Int 2016; 36:1783-1792. doi:10.1111/liv.13177.

[106] Maden CH, Fairman D, Chalker M, Costa MJ, Fahy WA, Garman N, et al Safety, tolerability and pharmacokinetics of GSK3008348, a novel integrin av $\beta 6$ inhibitor, in healthy participants. Eur J Clin Pharmacol 2018;74:701709. doi:10.1007/s00228-018-2435-3.

[107] Traber PG, Chou H, Zomer E, Hong F, Klyosov A, Fiel MI, et al. Regression of fibrosis and reversal of cirrhosis in rats by galectin inhibitors in thioacetamide-induced liver disease. PLoS One 2013;8:e75361. doi:10.1371/ journal.pone.0075361.

[108] Harrison SA, Marri SR, Chalasani N, Kohli R, Aronstein W, Thompson GA et al. Randomised clinical study: GR-MD-02, a galectin-3 inhibitor, vs. placebo in patients having non-alcoholic steatohepatitis with advanced fibrosis. Aliment Pharmacol Ther 2016;44:1183-1198. doi:10.1111/apt.13816.

[109] Harrison SA, Dennis A, Fiore MM, Kelly MD, Kelly CJ, Paredes AH, et al. Utility and variability of three non-invasive liver fibrosis imaging modalities to evaluate efficacy of GR-MD-02 in subjects with NASH and bridging fibrosis during a phase-2 randomized clinical trial. PLoS One 2018;13:e0203054 doi: $10.1371 /$ journal.pone.0203054

[110] Chalasani N, Abdelmalek MF, Garcia-Tsao G, Vuppalanchi R, Alkhouri N, Rinella $M$, et al. Effects of belapectin, an inhibitor of galectin-3, in patients with nonalcoholic steatohepatitis with cirrhosis and portal hypertension. Gaswith nonalcoholic steatohepatitis with cirrhosis and portal hypertension. Gas-
troenterology 2020;158:1334-1345.e5. doi:10.1053/j.gastro.2019.11.296.

[111] Marra F, Tacke F. Roles for chemokines in liver disease. Gastroenterology 2014;147:577-594.e1. doi:10.1053/j.gastro.2014.06.043.

[112] Parker R, Weston CJ, Miao Z, Corbett C, Armstrong MJ, Ertl L, et al. CC chemokine receptor 2 promotes recruitment of myeloid cells associated with insulin resistance in nonalcoholic fatty liver disease. Am J Physiol Gastrointest Liver Physiol 2018;314:G483-G493. doi:10.1152/ ajpgi.00213.2017.

[113] Seki E, De Minicis S, Gwak GY, Kluwe J, Inokuchi S, Bursill CA, et al. CCR1 and CCR5 promote hepatic fibrosis in mice. J Clin Invest 2009;119:18581870. doi: $10.1172 /$ jci37444.

[114] Friedman SL, Ratziu V, Harrison SA, Abdelmalek MF, Aithal GP, Caballeria $\mathrm{J}$, et al. A randomized, placebo-controlled trial of cenicriviroc for treatment of nonalcoholic steatohepatitis with fibrosis. Hepatology 2018;67:17541767. doi:10.1002/hep.29477.

[115] Ratziu V, Sanyal A, Harrison SA, Wong VW, Francque S, Goodman Z, et al. Cenicriviroc treatment for adults with nonalcoholic steatohepatitis and fibrosis: Final analysis of the phase $2 b$ CENTAUR study. Hepatology 2020;72:892-905. doi:10.1002/hep.31108.

[116] Anstee OM, Neuschwander-Tetri BA, Wong VW, Abdelmalek MF, Younossi ZM, Yuan J, et al. Cenicriviroc for the treatment of liver fibrosis in adults ZM, Yuan J, et al. Cenicriviroc for the treatment of liver fibrosis in adults
with nonalcoholic steatohepatitis: AURORA Phase 3 study design. Contemp with nonalcoholic steatohepatitis: AURORA Phase 3 study design
Clin Trials 2020;89:105922. doi:10.1016/j.cct.2019.105922.

[117] Weston CJ, Shepherd EL, Claridge LC, Rantakari P, Curbishley SM, Tomlinson JW, et al. Vascular adhesion protein-1 promotes liver inflammation and drives hepatic fibrosis. J Clin Invest 2015;125:501-520. doi:10.1172/ JCI73722.

[118] Konerman MA, Jones JC, Harrison SA. Pharmacotherapy for NASH: Current and emerging. J Hepatol 2018;68:362-375. doi:10.1016/j.jhep.2017. rent and 10.015 .

[119] Boehringer Ingelheim discontinues development of BI 1467335 for NASH. Available from: https://www.firstwordpharma.com/node/1688715. 